\title{
Designing Vehicle Turning Restrictions Based on the Dual Graph Technique
}

\author{
Lihui Zhang, ${ }^{1}$ Hongsheng Qi, ${ }^{1}$ Dianhai Wang, ${ }^{1}$ Zhong Wang, ${ }^{2}$ and Jian Yang ${ }^{2}$ \\ ${ }^{1}$ College of Civil Engineering and Architecture, Zhejiang University, 866 Yuhangtang Road, Hangzhou 310058, China \\ ${ }^{2}$ School of Transportation and Logistics, Dalian University of Technology, No. 2, Linggong Road, Dalian, China \\ Correspondence should be addressed to Hongsheng Qi; qihongsheng@zju.edu.cn
}

Received 23 November 2016; Accepted 12 March 2017; Published 6 April 2017

Academic Editor: Salvatore Alfonzetti

Copyright (c) 2017 Lihui Zhang et al. This is an open access article distributed under the Creative Commons Attribution License, which permits unrestricted use, distribution, and reproduction in any medium, provided the original work is properly cited.

\begin{abstract}
This paper investigates the turning restriction design problem that optimizes the turning restriction locations so as to minimize the total system travel time under the assumption of asymmetric user equilibrium. We first transform a transportation network into a dual graph, where traffic turning movements are explicitly modeled as dual links. The dual transformation allows us to derive a link-based formulation for the turning restriction design problem. Asymmetric user equilibrium is incorporated in the model as a set of nonlinear constraints. A dual-based heuristic algorithm is employed to solve the problem, by sequentially solving a relaxed turning restriction design problem and a design updating problem.
\end{abstract}

\section{Introduction}

Heavy turning traffic can bring challenges to traffic control at road intersections and may even cause severe congestion at the network level if not treated properly. One way to handle heavy turns is to impose restrictions, so that the turning vehicles need to take alternative routes to achieve their trips. The rerouting of the vehicles finally leads to the redistribution of traffic flow over the network and thus may have potential of reducing the overall network delay.

Take the Braess's network [1-4] shown in Figure 1 as an example, which is assumed to have five links already $a, b, c, d, e . b t$ and $b x$ are used to denote the link travel time and link flow for Braess's network. The link performance functions for the five links are $b t_{a}=10 \cdot b x_{a}, b t_{b}=50+b x_{b}$, $b t_{c}=50+b x_{c}, b t_{d}=10 \cdot b x_{d}$, and $b t_{e}=10+b x_{e}$. With a total demand of 6 , the system travel time at equilibrium is 552 . If a turning restriction from link $a$ to link $e$ is imposed, then, at the new equilibrium, the system travel time will reduce to 498 with the same demand level. To be noted, the result is obtained due to the so-called Braess's Paradox, which may not always happen if links possess other forms of cost functions and if the demand level increases [5].
This paper investigates the turning restriction design problem (TRDP), to optimally select the locations to impose turning restrictions, so as to improve the overall network performance. The research on TRDP in the literature is rather limited. Recently, Long et al. [6] formulated the TRDP into a bilevel programming problem. The upper-level problem decides where turning restrictions are to be imposed, with flow variables obtained from the lower-level stochastic user equilibrium problem. The decision of a turning restriction is modeled using a binary variable, and when a restriction is imposed, an extraordinary large cost will be added to all the paths utilizing this turn, so that these paths will rarely be selected in the new stochastic user equilibrium. The final model obtained is a mixed-integer nonlinear bilevel programming problem. They developed different sensitivity analysis-based solution algorithms (see, e.g., [7, 8]) to solve the complex problem. Since path information is needed in their modeling procedure to incorporate turning cost, they first applied a combination of the link elimination method [9] and Dial's [10] STOCH method to generate a path set and then employed the method of successive weighted averages [11] to solve the lower-level problem. Their numerical test on Sioux Falls network shows that turning restriction can 


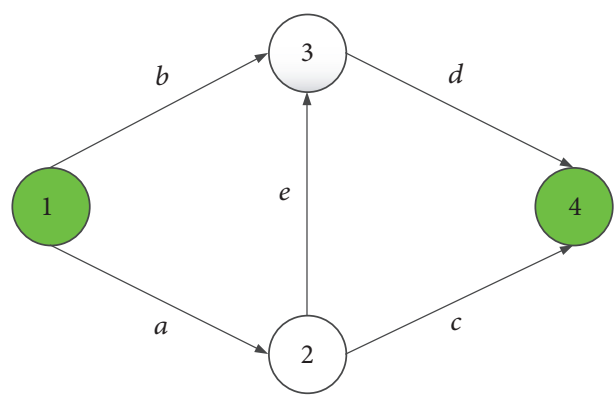

Figure 1: Braess's network.

effectively reduce the system travel cost, and the optimal turning restriction schemes are robust to the information and demand uncertainty. Later, Long et al. [12] expanded the model to further minimize the cost of total vehicle emissions, and the final model is biobjective and bilevel. They applied an artificial bee colony algorithm [13] to search for Paretooptimal turning restriction schemes. Numerical test shows that turning restriction can reduce the total system travel time and the cost of total vehicle emissions, especially during congestion period. Foulds et al. [14] applied the digraph construction process developed by Potts and Oliver [15] to model turning possibilities at intersections and developed another route-based formulation for TRDP. They applied an approximate solution algorithm named successive linear approximation method [16, 17] to solve the problem, accompanied by the column generation technique [18] to generate the path set between each OD pair. They further used multistart method [19] to improve the solution quality.

In this paper, we try to develop an alternative procedure to optimize the turning restriction locations. We improve the dual graph technique introduced by Añez et al. [20] to represent a target prime network, where each traffic movement, including the traffic turns, can be explicitly modeled as a dual link in the dual graph. The transformation is flexible in that it can be applied to the entire network or only to a portion of the network. The size of the dual graph is comparable to the graph generated by Potts and Oliver [15]. One benefit of the dual transformation is that we can directly formulate the TRDP in the same way as the classical network design problem (NDP). A turning restriction in the prime network is equivalent to a link deletion in the dual graph and thus can be modeled using a single binary variable. Since the decision variables are discrete, the final formulation obtained is more similar to the discrete network design problem (DNDP). Another benefit of the dual transformation is that path information is not required to incorporate turning cost, which brings the possibility of applying link-based solution algorithms to solve the TRDP. In the literature, various solution frameworks have been proposed to solve DNDP, for example, branch-and-bound technique [21], active-set algorithm [22] system-optimal-relaxation based method, and user-equilibrium-reduction based method [23], which are all link-based and may be employed to solve TRDP. This paper formulates TRDP as a single-level mixed-integer nonlinear program and then utilizes the active-set algorithm proposed by Zhang et al. [22] to solve for the optimal turning restriction designs.

The main contributions of the paper are first summarized as follows:

(1) The dual graph technique introduced by Añez et al. [20] is improved to explicitly model the turning restrictions on general transportation networks;

(2) An explicit link-based formulation is derived for TRDP, so that no path information is required to solve the problem;

(3) The derived TRDP formulation is in line with those for DNDP, so that the solution algorithms for DNDP can be applied to solve TRDP;

(4) Asymmetric user equilibrium is transformed into a set of nonlinear constraints, so that the TRDP formulation is a single-level program;

(5) Numerical tests on two networks are performed to demonstrate the proposed solution procedure.

The remainder of the paper is organized as follows: the dual graph technique is first introduced in the next section, accompanied by a short example. Section 3 delivers the detailed procedure to obtain the link-based formulation for TRDP, and Section 4 briefly introduces the active-set technique that incorporates commercial solvers to solve the TRDP. Numerical examples on two networks are presented in Section 5 to validate the proposed solution procedure. Concluding remarks are drawn in the last section.

\section{Network Representation}

The dual graph technique was claimed to be a powerful way to represent networks when turning and/or transfer movements were considered [20]. The dual graph is built according to the known prime network, which is denoted as $G(N, M)$, where $N$ and $M$ are the sets of nodes and links, respectively. Each link is either represented as a single letter $a$ or node pair $(i, j)$, where $i \neq j$ and $i, j \in N$.

A traffic movement, starting from one link and ending at the other, can be represented as a dual link, as the dashed link $(e, f)$ in Figure 2. In the prime network, a traffic movement can be represented as a triplet $(i, j, k)$ to indicate that the flow is from link $(i, j)$ to link $(j, k)$; subsequently, the set containing all traffic movements including the turning movements can be represented as follows:

$$
T=\{(i, j, k):(i, j) \in M,(j, k) \in M\} .
$$

Note that, when $i=k$, the corresponding movement is a $\mathrm{U}$ turn.

The dual graph is denoted as $\bar{G}(\bar{N}, \bar{M})$, where $\bar{N}$ and $\bar{M}$ are the sets of nodes and links in the dual graph. $\bar{N}$ contains two portions; one portion includes the centroids in the prime network (like node $j$ ) and the other portion includes the dual nodes representing all the links in the prime network (like nodes $e$ and $f$ ). $\bar{M}$ contains the dual links connecting the dual nodes and also connecting the centroids to the nearest dual nodes. The dual transformation can be performed automatically with some computer program. Once transformed, the dual graph will not change during our 


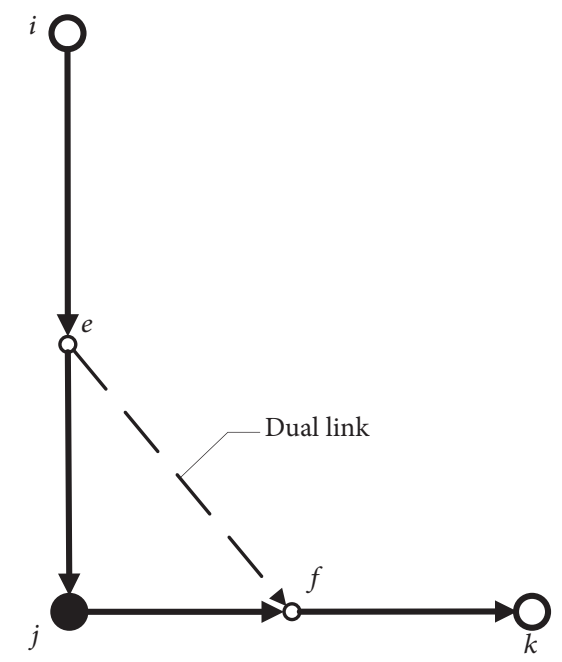

FIGURE 2: Illustration of a dual link.

solution procedure, so the transformation manipulation itself will not bring too much computation burden.

Figure 3 gives an example of transforming a small network. In the prime network (a), nodes 11 and 14 are centroids, and node 23 is an intersection. Following Añez et al. [20], the dual graph (b) can be obtained. The centroids 11 and 14 remain in the dual graph, and the intersection node 23 is not kept. Since all the links in (a) are represented as dual nodes in (b), 16 dual nodes are created in (b). The red links are those representing turning movements, and the arcs are for through traffic. Centroids generate demand or attract demand and thus need to be connected with dual nodes around them.

Direct assigning demand on the dual graph obtained by following Añez et al. [20] may not obtain the same flow distribution as on the prime network, since the network transformation will change the path sets that are feasible to the travelers. A bunch of redundant paths are generated, while the paths with U-turn at some road intersections are eliminated. Take the right turn $124 \rightarrow 40$ in Figure 3(b), for example; when it is prohibited, vehicles can still make right turn by using path $124 \rightarrow 14 \rightarrow 40$ or more complicatedly make U-turn at node 11 by using path $124 \rightarrow 121 \rightarrow 11 \rightarrow$ $38 \rightarrow 40$. While the U-turn options at centroids 11 and 14 are kept, the U-turn options at intersection 23 are eliminated. Thus dual graph (b) cannot serve our target to restrict turning movements, since the resulting flow distribution will probably be different from the equilibrium flow distribution on the prime network.

In reality, with many turning restrictions imposed, Uturn is a common choice of travelers to finish their trips. We further modify graph (b) by introducing a bunch of dummy nodes to replace the nodes in the prime network, as shown in graph (c). For each centroid, the number of dummy nodes is equal to its indegree/outdegree. Take node 14, for example; three dummy nodes $14-1,14-2$, and $14-3$ are created, to eliminate the path $124 \rightarrow 14 \rightarrow 40$, while the U-turn option or the path $124 \rightarrow 121 \rightarrow 11-3 \rightarrow 38 \rightarrow 40$ remains available for these travelers. The introduction of dummy nodes prevents travelers from selecting redundant paths; thus traffic assignment on dual graph (c) will produce the same flow distribution as on the prime network (a).

\section{Formulation of the Turning Restriction Design Problem}

3.1. Link Travel Cost of Dual Graph. Since the dual links are essentially virtual links, explicit travel time functions like those in the form of Bureau of Public Road (BPR) function cannot be applied directly. We derive the dual link travel cost based on the link travel cost in the prime network. $v$ and $x$ denote the link flow vectors in the prime and dual graphs, respectively. For simplicity, we assume that the dual nodes are all located in the middle points of the original prime links; then, the cost to make a turn $(i, j, k)$ or to traverse the corresponding dual link can be obtained as follows:

$$
c_{i j k}=0.5 \cdot\left(t_{i j}+t_{j k}\right) \text {. }
$$

The travel cost on the dual link equals half of the travel times on the starting and ending links. Travel cost of other types of dual links, like the through links, can be derived similarly.

The link travel time in the prime network is normally assumed to only depend on its link volume or

$$
t_{i j}=t_{i j}\left(v_{i j}\right) .
$$

Then the travel costs of the dual links can be expressed as a function of the flow distribution on the prime network or

$$
c=F(v) \text {. }
$$

On the other hand, according to the topological relationship between the prime and dual graphs, the link flow in the prime network can be derived based on the link flow in the dual graph. For example, in Figure 3, $v_{23,14}=x_{48,124}+x_{130,124}$ holds. We use $G$ to denote the flow relationship or

$$
v=G(x) .
$$

To be noted, $G$ is not invertible. Then, the link travel cost on the dual graph can be expressed as follows:

$$
c(x)=F(G(x)) \text {. }
$$

The decisions of turning restrictions are modeled using a set of binary variables $\beta_{i j},(i, j) \in \bar{M}$. $\beta_{i j}$ equals 0 means the corresponding turning movement is prohibited; otherwise vehicles can make turn here. When a link is not a turning link, restriction will never be imposed, so $\beta_{i j}$ always equals 1. To incorporate the information of turning restrictions, we further modify the travel cost function of the dual links:

$$
c_{i j}(x, \beta)=f(g(x))+\left(1-\beta_{i j}\right) \cdot \mathrm{BN},
$$

where $\mathrm{BN}$ is a big number. When a turning movement is restricted, a large cost will be added to the dual link so that travelers will divert to other paths. 


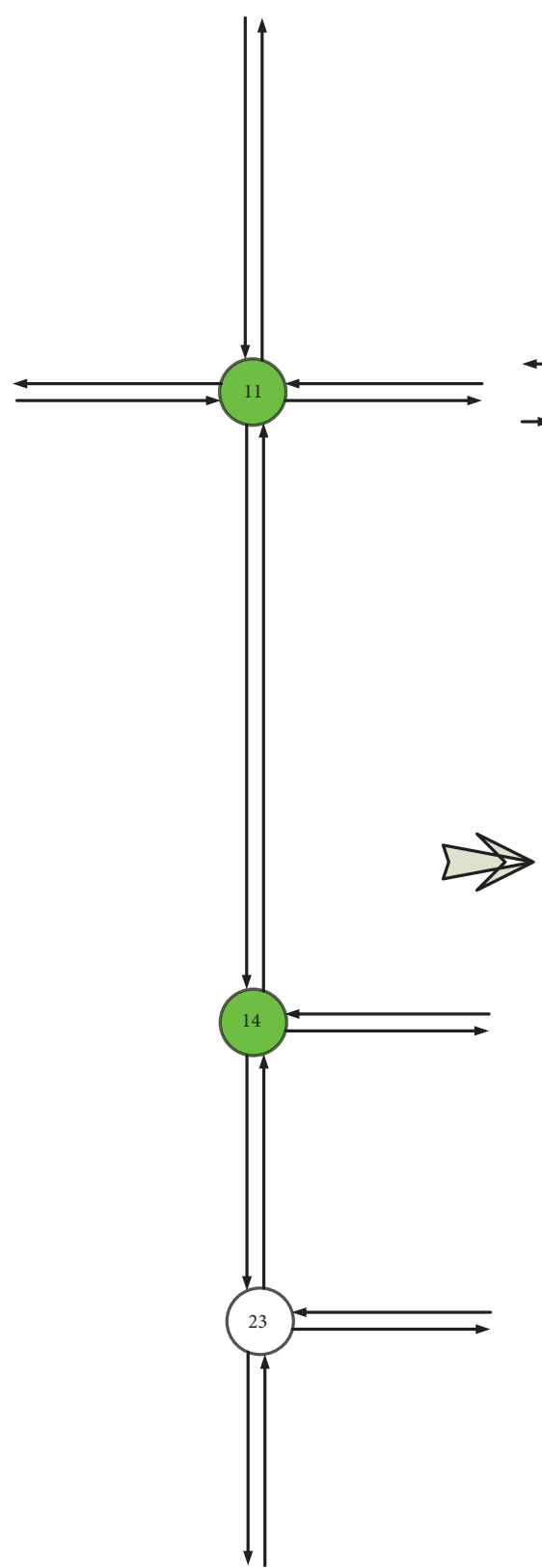

(a)

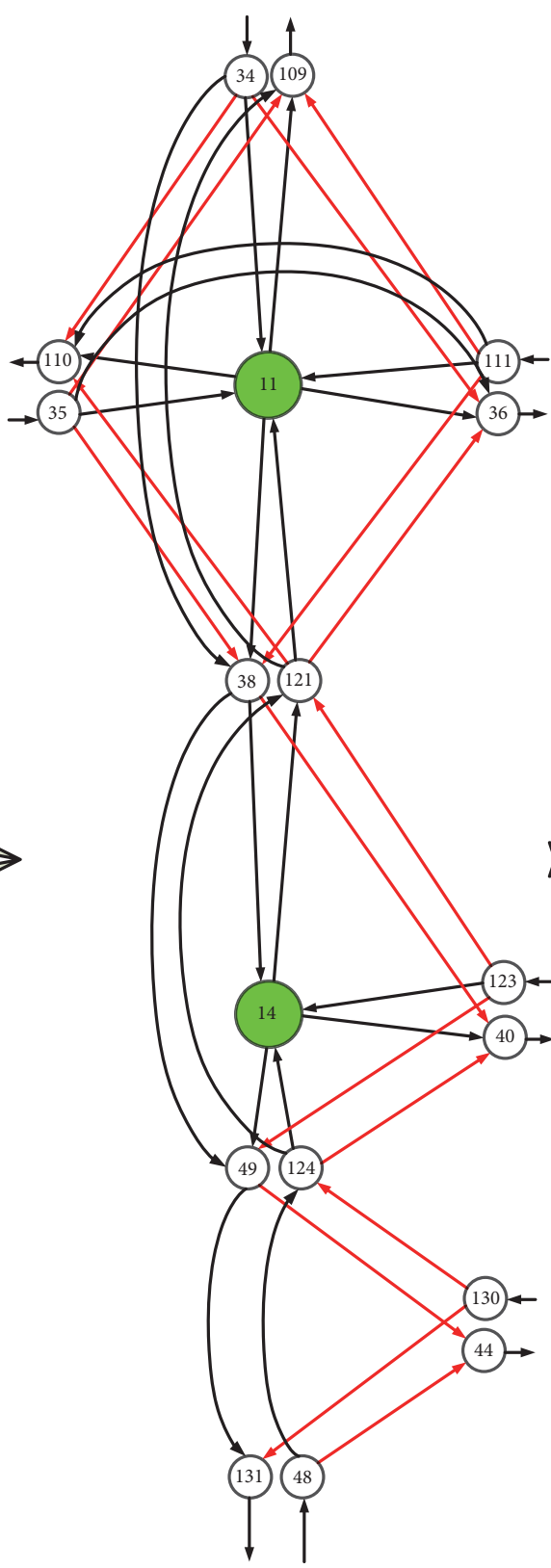

(b)

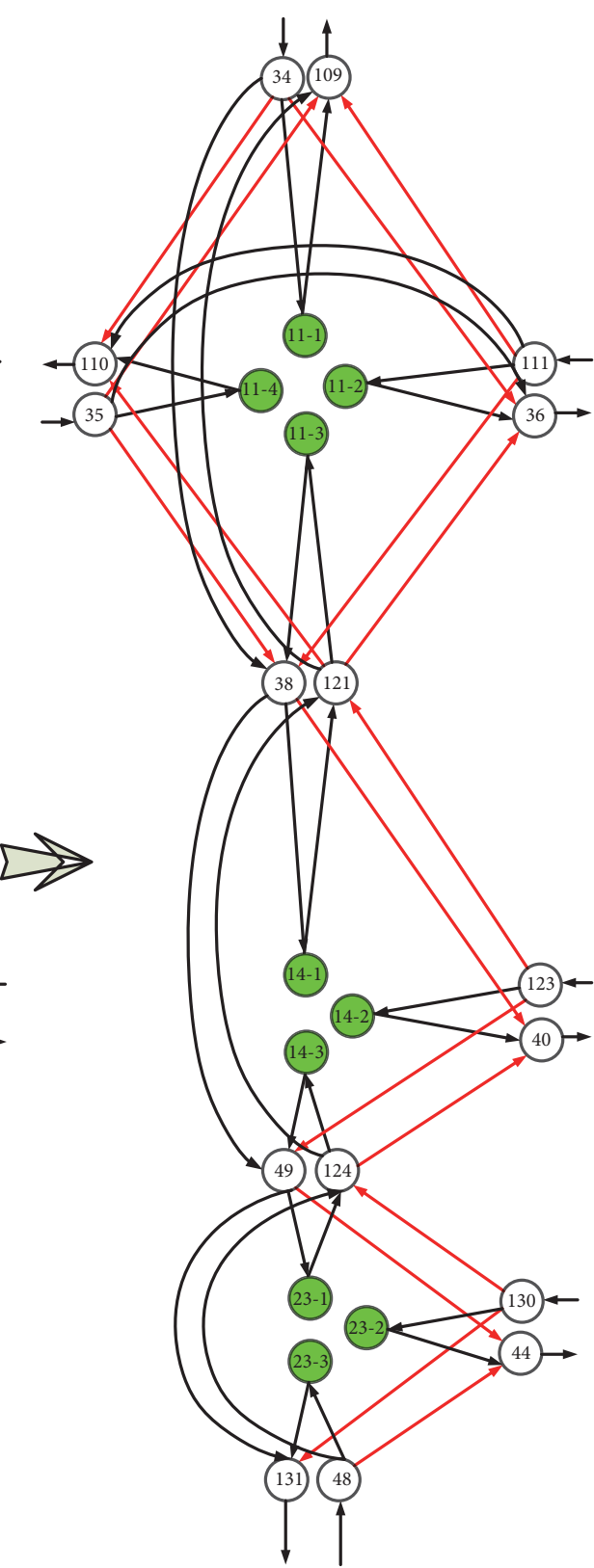

(c)

FIgURE 3: Dual transformation of an example network.

3.2. Bilevel Model for TRDP. It is assumed that travel demand is given and fixed. Let $W$ denote the set of OD pairs. For each OD pair $w \in W$, the travel demand is denoted as $d^{w}$. Then the feasible flow distributions in the dual graph can be expressed as follows:

$$
\begin{aligned}
& x=\sum_{w} x^{w}, \\
& \sum_{\forall j, i j \in \bar{M}} x_{i j}^{w}-\sum_{\forall j, j i \in \bar{M}} x_{j i}^{w}= \begin{cases}d^{w} & i=\text { origin } \\
-d^{w} & i=\text { destination } \\
0 & i=\text { others }\end{cases}
\end{aligned}
$$

$$
x^{w} \geq 0 \quad \forall w
$$

Equation (9) is the flow conservation constraints and (10) is nonnegative constraints of traffic flow.

Different turning restriction designs influence different group of travelers and may lead to quite different equilibrium flow distributions. Thus, it is natural to formulate the TRDP into a bilevel structure to take travelers' route choice behavior into account. The upper-level problem optimizes the turning restriction design by maximizing some system performance measure from the perspective of decision makers. For simplicity, we select the total system travel time (TTT) as the 
upper-level objective function; then, the problem can be described as follows:

$$
\begin{array}{ll}
\min & c(x, \beta)^{T} x \\
\text { s.t. } & \beta_{i j}=1 \quad \forall(i, j) \in \bar{M} \backslash \bar{T} \\
& \beta_{i j}=\{0,1\} \quad \forall(i, j) \in \bar{T},
\end{array}
$$

where $\bar{T}$ is the set of all candidate turning links. Given the turning restriction design, the flow variable $x$ in the upperlevel problem is obtained by solving the lower-level user equilibrium (UE) problem. For the UE problem, as the link travel cost functions derived as in (7) are asymmetric, equivalent mathematical program in a form similar to the Beckmann-McGuire-Winsten (BMW) formulation [24] cannot be obtained. However, it can be generally written in form of a variational inequality problem (VI):

$U E-V I$

$$
\begin{array}{ll} 
& c\left(x^{*}, \beta\right)^{T}\left(x-x^{*}\right) \geq 0 \\
\text { s.t. } \quad x=\sum_{w} x^{w} & \\
\sum_{\forall j, i j \in \bar{M}} x_{i j}^{w}-\sum_{\forall j, j i \in \bar{M}} x_{j i}^{w}= \begin{cases}d^{w} & i=\text { origin } \\
-d^{w} & i=\text { destination } \\
0 & i=\text { others } \quad \forall i, \forall w \\
x^{w} \geq 0 \quad \forall w .\end{cases}
\end{array}
$$

3.3. Single-Level Model for TRDP. We employ the technique developed in Aghassi et al. [25] to avoid directly solving the VI problem. According to the duality theory, the following nonlinear optimization problem (UE-NLP) is formulated:

\section{$U E-N L P$}

$$
\begin{aligned}
& \min \sum_{i j} c_{i j}\left(x, \beta_{i j}\right) \cdot x_{i j}-\sum_{w} \sum_{i} \rho_{i}^{w} \cdot d_{i}^{w} \\
& \text { s.t. } \quad x=\sum_{w} x^{w} \\
& \sum_{\forall j, i j \in \bar{M}} x_{i j}^{w}-\sum_{\forall j, j i \in \bar{M}} x_{j i}^{w}
\end{aligned}
$$

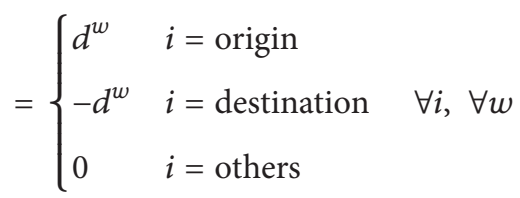

$$
\begin{aligned}
& x^{w} \geq 0 \quad \forall w \\
& \rho_{i}^{w}-\rho_{j}^{w} \leq c_{i j}\left(x, \beta_{i j}\right) \quad \forall(i, j) \in \bar{M}, \forall w,
\end{aligned}
$$

where $\rho_{i}^{w}$ is the Lagrangian variable associated with flow conservation constraints (9), which can be regarded as node potential. According to the strong duality theory, when a solution leads to a duality gap of zero, then the solution must be optimal to both the prime and dual problems. Parallelly, if the optimal objective value of UE-NLP is zero, then it can be claimed that the resulting link flow distribution is in user equilibrium or satisfies UE-VI. With given turning restriction design, the above formulation is a nonlinear program and can be solved with commercial solvers without difficulty. Furthermore, since the equilibrium conditions can be represented by the optimality condition of the NE-VI, we can convert the bilevel problem into a single-level problem as follows:

TRDP

$$
\begin{aligned}
& \min \sum_{i j} c_{i j}\left(x, \beta_{i j}\right) \cdot x_{i j} \\
& \text { s.t. } \quad x=\sum_{w} x^{w} \\
& \sum_{\forall j, i j \in \bar{M}} x_{i j}^{w}-\sum_{\forall j, j i \in \bar{M}} x_{j i}^{w} \\
& = \begin{cases}d^{w} & i=\text { origin } \\
-d^{w} & i=\text { destination } \quad \forall i, \forall w \\
0 & i=\text { others }\end{cases} \\
& x^{w} \geq 0 \quad \forall w \\
& \sum_{i j} c_{i j}\left(x, \beta_{i j}\right) \cdot x_{i j}-\sum_{w} \sum_{i} \rho_{i}^{w} \cdot d_{i}^{w}=0 \quad \forall i, j \\
& \rho_{i}^{w}-\rho_{j}^{w} \leq c_{i j}\left(x, \beta_{i j}\right) \quad \forall(i, j) \in \bar{M}, \forall w \\
& \beta_{i j}=1 \quad \forall(i, j) \in \bar{M} \backslash \bar{T} \\
& \beta_{i j}=\{0,1\} \quad \forall(i, j) \in \bar{T} .
\end{aligned}
$$

The models proposed in Long et al. [6] and Foulds et al. [14] are both path-based. Actually no explicit formulations are presented in both papers, due to the difficulty in explicitly writing the constraints for turning restrictions. Their models are both bilevel models, with the upper-level problems deciding where to impose the turning restrictions and lowerlevel problems describing the travelers' route choice behavior, while the above TRDP formulation is an explicit one-level mixed-integer nonlinear programming problem (MINLP), with the constraints for turning restrictions embedded.

\section{Solving the Turning Restriction Design Problem}

One approach to solve MINLP is to first linearize the nonlinear portions in the formulation so as to transform MINLPs into mixed-integer linear programming problems (MIPs), and then apply techniques for MIP to solve for optimal solutions (see, e.g., $[26,27])$. The other approach first deals with the integer variables contained in MINLP formulations so as 
to transform MINLPs into NLPs, and then apply commercial solvers for NLP to solve for the final solutions. We here employ the active-set algorithm (see, e.g., [28, 29]) to solve the TRDP, which belongs to the second category.

The algorithm sequentially solves two subproblems: a relaxed TRDP (R-TRDP) given certain turning restriction design and an updating problem to update the restriction (UPDATE). The two problems are first given as follows:

\section{R-TRDP}

$$
\begin{aligned}
& \min \sum_{i j} c_{i j}\left(x, \beta_{i j}\right) \cdot x_{i j} \\
& \text { s.t. } \quad x=\sum_{w} x^{w} \\
& \sum_{\forall j, i j \in \bar{M}} x_{i j}^{w}-\sum_{\forall j, j i \in \bar{M}} x_{j i}^{w} \\
& = \begin{cases}d^{w} & i=\text { origin } \\
-d^{w} & i=\text { destination } \quad \forall i, \forall w \\
0 & i=\text { others }\end{cases} \\
& x^{w} \geq 0 \quad \forall w \\
& \sum_{i j} c_{i j}\left(x, \beta_{i j}\right) \cdot x_{i j}-\sum_{w} \sum_{i} \rho_{i}^{w} \cdot d_{i}^{w}=0 \quad \forall i, j \\
& \rho_{i}^{w}-\rho_{j}^{w} \leq c_{i j}\left(x, \beta_{i j}\right) \quad \forall(i, j) \in \bar{M}, \forall w \\
& \beta_{i j}=1 \quad \forall(i, j) \in \bar{M} \backslash \bar{T} \\
& \beta_{i j}=0 \quad \forall(i, j) \in \Omega_{0} \\
& \beta_{i j}=1 \quad \forall(i, j) \in \Omega_{1} .
\end{aligned}
$$

\section{UPDATE}

$$
\begin{array}{ll}
\min & \sum_{i j} \lambda_{i j} \beta_{i j} \\
\text { s.t. } & \sum_{i j}\left[\left(1-\widehat{\beta}_{i j}\right) \beta_{i j}+\widehat{\beta}_{i j}\left(1-\beta_{i j}\right)\right] \geq 1 \quad \forall \widehat{\beta}_{i j} \in \phi \\
& \beta_{i j} \in\{0,1\} \quad \forall(i, j) \in \bar{T} .
\end{array}
$$

In R-TRDP, given a certain turning restriction design, the $\beta_{i j}$ value for each candidate link is fixed. $\Omega_{0}$ and $\Omega_{1}$ are two sets containing all links indices with $\beta_{i j}$ equal to 0 or 1 . The two sets are mutually exclusive, and $\Omega_{0} \cup \Omega_{1}=\bar{T}$. With fixed $\beta_{i j}$ value, the decision variables are all continuous in R-TRDP. Then, R-TRDP becomes a regular NLP, which can be directly solved with commercial solvers. If the problem can be solved to optimality, then the constraints can ensure that the resulting flow distribution is in UE state. R-TRDP serves the purpose of evaluating the effectiveness of each turning restriction scheme by minimizing the total system cost and at the mean time provides us with clues to search for better design schemes.
The UPDATE problem is designed to search for new restriction designs that may improve the system performance. $\lambda_{i j}$ is the dual multipliers associated with $\beta_{i j}$ in RTRDP. By solving the nonlinear R-TRDP, $\lambda_{i j}$ can be obtained. UPDATE has the objective of maximizing the potential improvement can be made to the total system travel time. Each time a new restriction design is found, it will be fed into the R-TRDP to see if the system travel is reduced. Since the direct update between 0 and 1 is discrete, reduction of system travel time is not guaranteed. Thus new update will be performed to find another restriction design. To ensure previous restriction designs will not be generated again, constraint (16) is added to the search problem. $\phi$ is the set containing all the previous designs generated before an improvement design is found. If a new search finds a turning restriction design already in $\phi$, then the left hand side of constraint (16) will be 0 for that old scheme; thus the constraint is violated. In this way, constraint (16) will prevent old schemes generated again. $\phi$ will be cleared once an improvement design is obtained.

The whole solution procedure is described as follows.

Step 1. Set $t=1$; generate an initial restriction design $\beta^{1}$ or $\left(\Omega_{0}^{1}, \Omega_{1}^{1}\right)$.

Step 2. Solve the R-TRDP with $\left(\Omega_{0}^{t}, \Omega_{1}^{t}\right)$ to determine $\lambda^{t}$, the KKT multiplier of $\beta^{t}$, and objective function value $Z$. Set $Z^{t}=$ $Z$.

Step 3.

(a) Solve UPDATE with $\lambda^{t}$ for solution $\bar{\beta}$.

If the objective value equals 0 , then the optimal solution is found; stop the algorithm. Otherwise, go to Step 3(b).

(b) Update

$$
\begin{aligned}
& \widehat{\Omega}_{0}=\left(\Omega_{0}^{t}-\left\{(i, j) \in \Omega_{0}^{t}: \widehat{\beta}_{i j}=1\right\}\right) \\
& \cup\left\{(i, j) \in \Omega_{1}^{t}: \widehat{\beta}_{i j}=0\right\}, \\
& \widehat{\Omega}_{1}=\left(\Omega_{1}^{t}-\left\{(i, j) \in \Omega_{1}^{t}: \widehat{\beta}_{i j}=0\right\}\right) \\
& \cup\left\{(i, j) \in \Omega_{0}^{t}: \widehat{\beta}_{i j}=1\right\} .
\end{aligned}
$$

(c) Solve the R-TRDP with $\left(\widehat{\Omega}_{0}, \widehat{\Omega}_{1}\right)$ to obtain $\bar{\lambda}^{t}$ and $\bar{Z}$. If $\widehat{Z}<Z^{t}$, update $t=t+1$, let $\Omega_{0}^{t}=\widehat{\Omega}_{0}, \Omega_{1}^{t}=\widehat{\Omega}_{1}$, and $\lambda^{t}=\bar{\lambda}^{t}, \phi=\varnothing$; then go to Step 3(a). Otherwise, update $\phi=\phi \cup\{\widehat{\beta}\}$ and go to Step 3(a).

The algorithm terminates at Step 3(a), when no improvement design can be found. It has been proved that the algorithm will produce a strong stationary point in finite number of iterations [22].

\section{Numerical Test}

To demonstrate the proposed modeling approach, numerical tests are performed on two networks, an artificial six-node 


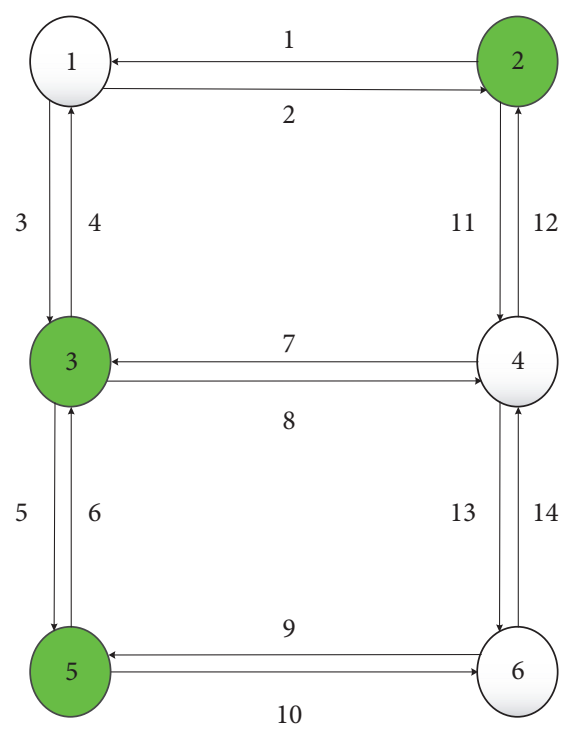

FIGURE 4: Six-node network.

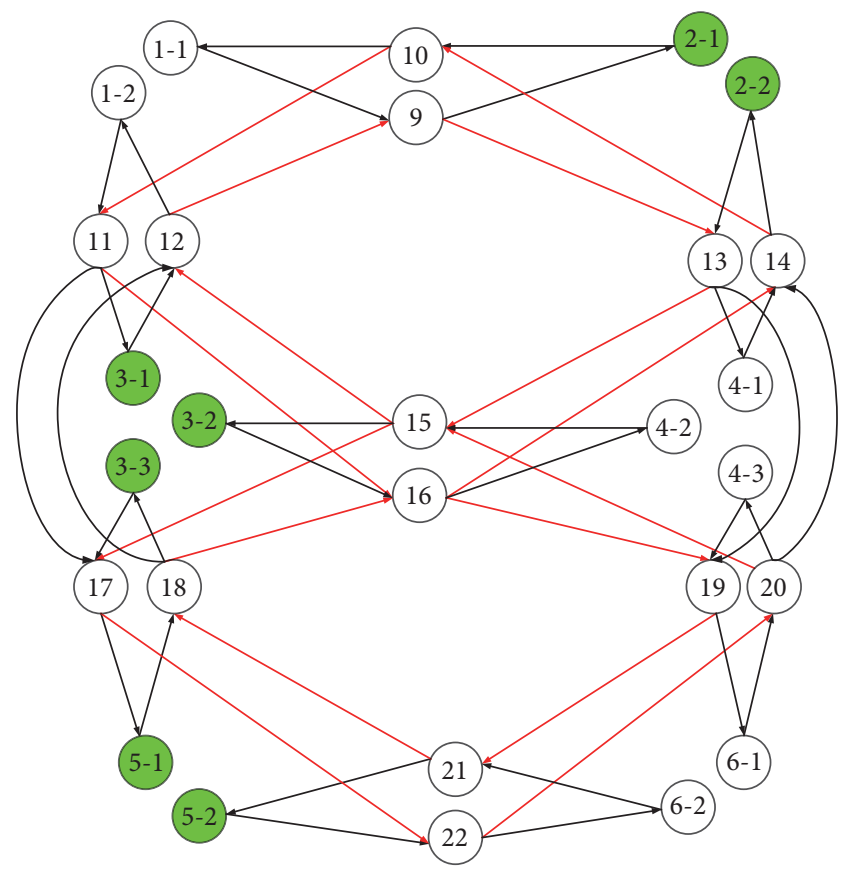

FIGURE 5: Dual graph of six-node network.

network and Sioux Falls network [21,30]. The results reported below are from our GAMS [31] implementation on a Lenovo computer with $3.4 \mathrm{GHz}$ Intel i7 CPU and $4 \mathrm{~GB}$ of Ram.

5.1. Six-Node Network. The prime network in Figure 4 is transformed into the improved dual graph in Figure 5. The prime network contains 6 nodes and 14 links, and nodes 2, 3, and 5 are centroids. The demand table is given in Table 1 . The size of the dual graph is larger, with 28 nodes (14 dual nodes, 14 dummy nodes) and 48 links.
TABLE 1: OD Table for six-node network.

\begin{tabular}{lccc}
\hline Origin & \multicolumn{3}{c}{ Destination } \\
\hline 2 & 2 & 3 & 5 \\
3 & 0 & 12.0 & 10.3 \\
5 & 12.0 & 0 & 24.0 \\
\hline
\end{tabular}

TABLE 2: Link attributors for six-node network.

\begin{tabular}{lcc}
\hline Link $a$ & $c_{a}$ & $t_{a}^{0}$ \\
\hline$(1,2)$ & 13.86 & 2.40 \\
$(2,1)$ & 9.90 & 2.40 \\
$(1,3)$ & 1.52 & 3.00 \\
$(3,1)$ & 20.00 & 3.00 \\
$(2,4)$ & 21.62 & 1.20 \\
$(4,2)$ & 9.80 & 1.20 \\
$(3,4)$ & 10.10 & 2.00 \\
$(4,3)$ & 10.10 & 2.00 \\
$(3,5)$ & 27.83 & 1.80 \\
$(5,3)$ & 27.83 & 1.80 \\
$(4,6)$ & 10.09 & 3.00 \\
$(6,4)$ & 10.09 & 3.00 \\
$(5,6)$ & 10.27 & 3.00 \\
$(6,5)$ & 10.27 & 3.00 \\
\hline
\end{tabular}

The BPR function used to calculate link travel time in the prime network is written as follows:

$$
t_{a}\left(v_{a}\right)=t_{a}^{0}\left(1+0.15\left(\frac{v_{a}}{c_{a}}\right)^{4}\right)
$$

where $t_{a}^{0}$ and $c_{a}$ are the free flow travel time and road capacity of link $a$. These link attributors are given in Table 2 .

Though the dual graph is around 4 times larger than the prime network, it does not bring much computation burden. A deterministic user equilibrium assignment typically takes around 0.1-0.2 computer second. We first check the UE assignment results from both the prime network and the dual network, to find whether their flow distributions are identical. We perform multiple tests on six-node network with different random initial solutions. Table 3 reports results from 20 runs, including the total system travel times from the 20 initial solutions and from the 20 optimal solutions, the improvements achieved, and the CPU times spent.

It can be observed that with different starting points, the final solutions achieve improvements roughly between $4 \%$ and $8 \%$ for six-node network. It generally takes more time to find the restriction schemes to reach objective value 365.177. Run 1 spends the largest amount of time. The detailed restriction designs are reported in Table 4 , where $\beta^{1}$ indicates the initial restriction scheme and $\beta^{n}$ indicates the optimal one generated. 0 in the cells means turning restriction is imposed on the link while 1 means no restriction. To further demonstrate the model and algorithm, Figure 6 shows the evolution of the objective function during the process to reach the optimal restriction scheme. 
TABLE 3: Numerical results for six-node network.

\begin{tabular}{|c|c|c|c|c|}
\hline Run & Initial TTT & Final TTT & Change (\%) & CPU time (s) \\
\hline 1 & 396.387 & 365.177 & 7.87 & 753 \\
\hline 2 & 396.387 & 366.109 & 7.64 & 142 \\
\hline 3 & 380.177 & 365.177 & 3.95 & 421 \\
\hline 4 & 382.670 & 365.177 & 4.57 & 482 \\
\hline 5 & 380.177 & 366.109 & 3.70 & 101 \\
\hline 6 & 396.387 & 366.109 & 7.64 & 60 \\
\hline 7 & 396.387 & 366.109 & 7.64 & 201 \\
\hline 8 & 396.387 & 366.109 & 7.64 & 82 \\
\hline 9 & 396.387 & 366.109 & 7.64 & 343 \\
\hline 10 & 396.387 & 366.109 & 7.64 & 166 \\
\hline 11 & 396.387 & 365.177 & 7.87 & 367 \\
\hline 12 & 380.177 & 365.177 & 3.95 & 623 \\
\hline 13 & 396.387 & 366.109 & 7.64 & 76 \\
\hline 14 & 396.387 & 365.177 & 7.87 & 184 \\
\hline 15 & 381.388 & 365.177 & 4.25 & 614 \\
\hline 16 & 381.388 & 366.109 & 4.01 & 100 \\
\hline 17 & 396.387 & 365.177 & 7.87 & 501 \\
\hline 18 & 381.388 & 365.177 & 4.25 & 386 \\
\hline 19 & 396.387 & 365.177 & 7.87 & 456 \\
\hline 20 & 396.387 & 365.177 & 7.87 & 279 \\
\hline
\end{tabular}

TABLE 4: One turning restriction design in six-node network.

\begin{tabular}{lcc}
\hline Link & $\beta^{1}$ & $\beta^{n}$ \\
\hline$(10,11)$ & 0 & 1 \\
$(12,9)$ & 1 & 1 \\
$(11,16)$ & 0 & 0 \\
$(15,12)$ & 0 & 1 \\
$(15,17)$ & 1 & 0 \\
$(18,16)$ & 1 & 0 \\
$(23,18)$ & 0 & 0 \\
$(17,24)$ & 1 & 0 \\
$(14,10)$ & 1 & 1 \\
$(9,13)$ & 1 & 1 \\
$(13,15)$ & 1 & 1 \\
$(16,14)$ & 1 & 1 \\
$(20,15)$ & 1 & 0 \\
$(16,19)$ & 0 & 0 \\
$(19,23)$ & 1 & 1 \\
$(24,20)$ & 1 & 1 \\
\hline
\end{tabular}

5.2. Sioux Falls Network. Sioux Falls network is widely adopted as test network in the transportation literature (see, e.g., $[6,12])$. We use the network attributors given in Zhang and Sun [32]. The prime network has 24 nodes and 76 links. After the transformation, the dual graph obtained contains 135 nodes and 360 links, among which 132 are turning links and are allowed to be restricted. A single deterministic user equilibrium assignment on the dual graph takes 1-2 seconds. Again, we first check the UE assignment results from both the prime network and the dual network, to find whether their

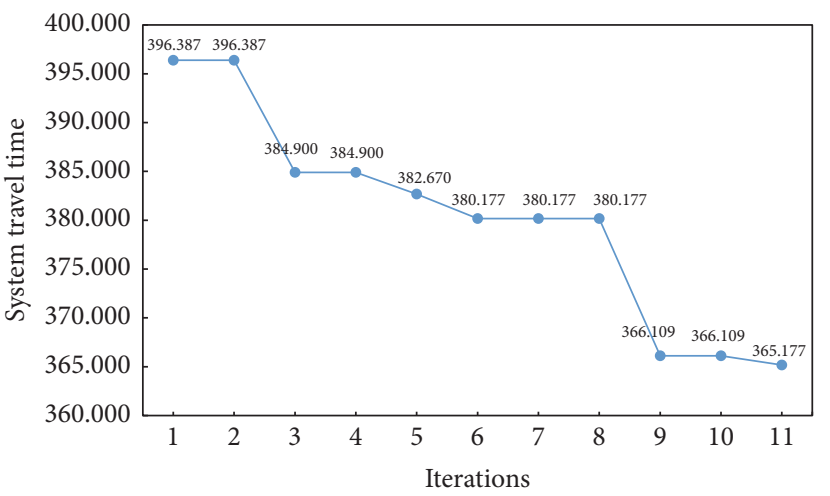

FIGURE 6: Evolution of objective value from six-node network.

flow distributions are identical, to guarantee the correctness of the network transformation. For a large network, the dual transformation may not be applied to the whole network, but only to selected intersections or arterials. For example, Figure 7 shows the transformation of only a portion of the prime network.

In our test, all the turning movements are allowed to be restricted. Figure 8 reports an optimal turning restriction design generated with a random initial solution. All the arrows shown in the figure are restricted turning movements. The algorithm takes 18720 computer seconds to reach the optimal solution. The total system travel time is reduced from 1359.672 to 1118.301 . Figure 9 shows the evolution of the objective value during the solution procedure.

By checking the detailed solution process for Sioux Falls network, we found that the majority of the computation time 


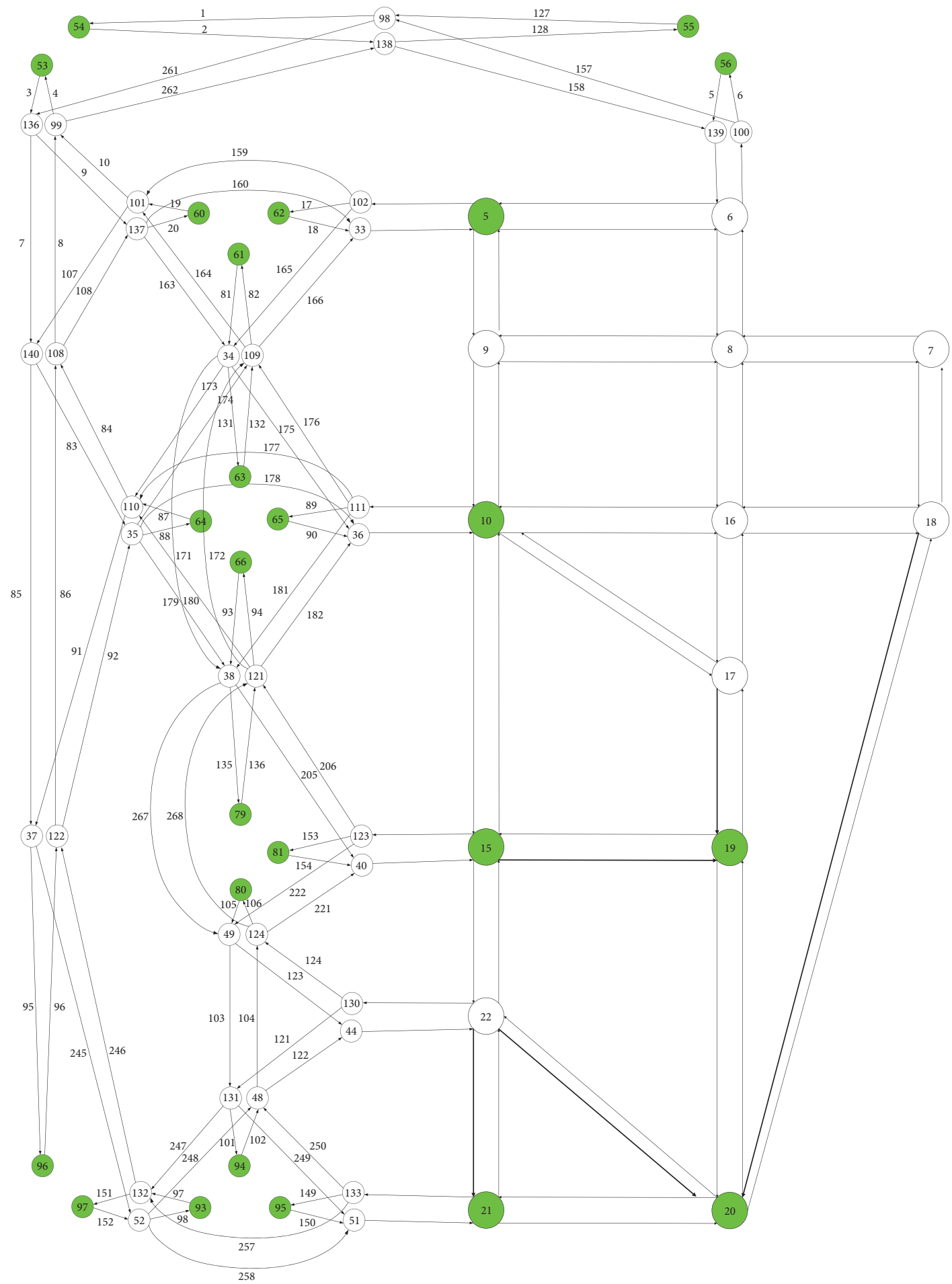

FIGURE 7: Dual graph of Sioux Falls network.

was consumed in verifying if a feasible solution leaded to some improvement in the objective function. Since all the turns can be restricted under the problem settings, the feasible solution set is very large, which leads to a large number of verification manipulations. Though the verification of one feasible solution only took 1-2 seconds, the total computation time is not short. However, for an offline planning problem like TRDP, 18720 seconds is still acceptable.

The active-set algorithm solves TRDP by sequentially solving some smaller-sized subproblems, which allows it to be applied for even larger networks; for example, Zhang et al. [22] successfully applied it on a network with 798 links, 


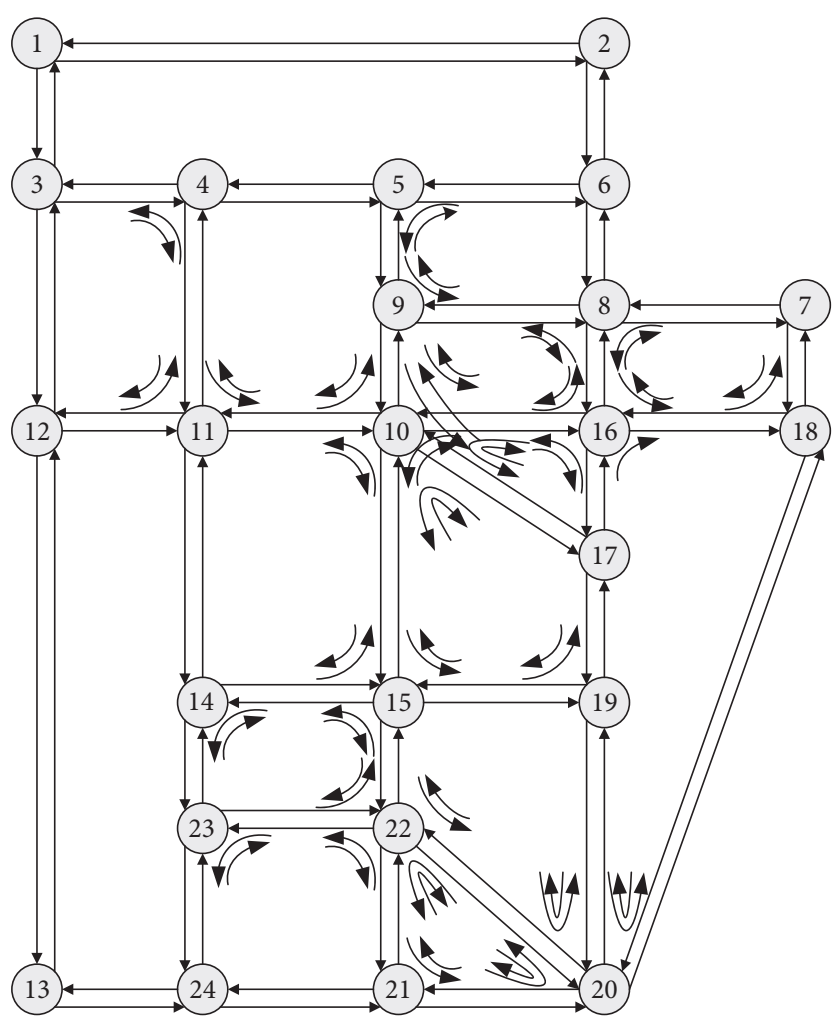

FIGURE 8: One turning restriction scheme in Sioux Falls Network.

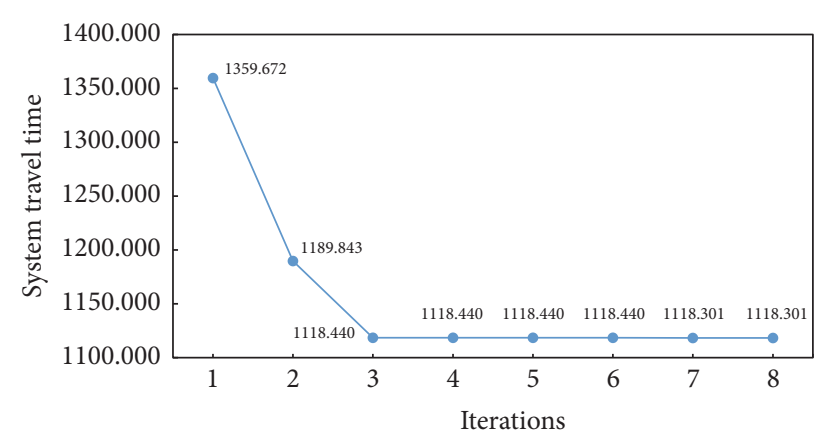

FIGURE 9: Evolution of objective value from Sioux Falls network.

501 nodes, and 158 OD pairs. For such larger networks, we can envision that the computation burden for solving TRDP will be high, if all turning movements can be restricted. A common approach to save computation time is to first set the candidate restriction points, based on field observations of traffic conditions or other practical criteria.

\section{Concluding Remarks}

This paper investigates the TRDP that tries to optimally select the locations to impose turning restrictions so as to improve the overall network performance. We propose an alternative approach that utilizes improved dual graph to explicitly model different traffic movements at intersections. After the dual transformation of the target network, the turning restriction design problem can be written into a link-based formulation, which allows us to employ link-based solution algorithms to solve the problem.

The first model obtained is a bilevel programming problem, with deterministic user equilibrium as the lower-level problem to capture the travelers' response to the upperlevel turning restriction decisions. We then transform the asymmetric user equilibrium problem into a regular nonlinear problem, to finally obtain a single-level mixed-integer nonlinear program for TRDP. The active-set algorithm is employed to solve the TRDP, which sequentially solves a relaxed TRDP and an updating problem. The active-set algorithm first deals with the large number of binary variables in the formulation, and then uses commercial NLP solvers to solve the problem. Numerical tests on two networks are performed to demonstrate the proposed solution procedure.

In our problem settings, we allow both right turn and leftturn traffic movements to be restricted, which can be relaxed to consider only left-turn restriction or can be strengthened to also consider restricting through traffic. The modeling approach is also flexible that the dual transformation can be applied to the whole network or only to a portion of the network when other practical constraints are considered.

\section{Conflicts of Interest}

The authors declare that they have no conflicts of interest.

\section{Acknowledgments}

This work was supported by the Key Science and Technology Innovation Team of Zhejiang Province under Grant [no. 2013TD09]; the Fundamental Research Funds for the Central Universities; and Lloyd's Register Foundation, which helps to protect life and property by supporting engineering-related education, public engagement, and the application of research.

\section{References}

[1] D. Braess, "Über ein paradoxon aus der verkehrsplanung," Unternehmenforschung, vol. 12, pp. 258-268, 1968.

[2] W. H. K. Lam, "Optimization of transport investment and pricing policies: the role of transport pricing in network design," Transportation Planning and Technology, vol. 13, no. 4, pp. 245258, 1989.

[3] E. I. Pas and S. L. Principio, "Braess' paradox: some new insights," Transportation Research Part B: Methodological, vol. 31, no. 3, pp. 265-276, 1997.

[4] A. Nagurney, "Congested urban transportation networks and emission paradoxes," Transportation Research Part D: Transport and Environment, vol. 5, no. 2, pp. 145-151, 2000.

[5] A. Nagurney, "The negation of the Braess paradox as demand increases: the wisdom of crowds in transportation networks," EPL, vol. 91, no. 4, Article ID 48002, 2010.

[6] J. Long, Z. Gao, H. Zhang, and W. Y. Szeto, "A turning restriction design problem in urban road networks," European Journal of Operational Research, vol. 206, no. 3, pp. 569-578, 2010. 
[7] J. Q. Ying and H. Yang, "Sensitivity analysis of stochastic user equilibrium flows in a bi-modal network with application to optimal pricing," Transportation Research Part B: Methodological, vol. 39, no. 9, pp. 769-795, 2005.

[8] J. Q. Ying, H. Lu, and J. Shi, "An algorithm for local continuous optimization of traffic signals," European Journal of Operational Research, vol. 181, no. 3, pp. 1189-1197, 2007.

[9] S. Bekhor, M. E. Ben-Akiva, and M. S. Ramming, "Route choice: choice set generation and probabilistic choice models," in Proceedings of the 4th TRISTAN Conference, Azores, Portugal, 2001.

[10] R. B. Dial, "A probabilistic multipath traffic assignment model which obviates path enumeration," Transportation Research, vol. 5, no. 2, pp. 83-111, 1971.

[11] H. X. Liu, X. He, and B. He, "Method of successive weighted averages (MSWA) and self-regulated averaging schemes for solving stochastic user equilibrium problem," Networks and Spatial Economics, vol. 9, no. 4, pp. 485-503, 2009.

[12] J. Long, W. Y. Szeto, and H.-J. Huang, "A bi-objective turning restriction design problem in urban road networks," European Journal of Operational Research, vol. 237, no. 2, pp. 426-439, 2014.

[13] D. Karaboga, "An idea based on honey bee swarm for numerical optimization," Technical Report TR06, Erciyes University, Engineering Faculty, Computer Engineering Department, Kayseri, Turkey, 2005.

[14] L. R. Foulds, D. C. Duarte, H. A. do Nascimento, H. J. Longo, and B. R. Hall, "Turning restriction design in traffic networks with a budget constraint," Journal of Global Optimization, vol. 60, no. 2, pp. 351-371, 2014.

[15] R. B. Potts and R. M. Oliver, Flows in Transportation Networks, Academic Press, London, UK, 1972.

[16] F. Palacios-gomez, L. Lasdon, and M. Engquist, "Nonlinear optimization by successive linear programming," Management Science, vol. 28, no. 10, pp. 1106-1120, 1982.

[17] H. D. Sherali, A. Narayanan, and R. Sivanandan, "Estimation of origin-destination trip-tables based on a partial set of traffic link volumes," Transportation Research Part B: Methodological, vol. 37, no. 9, pp. 815-836, 2003.

[18] G. B. Dantzig and P. Wolfe, "Decomposition principle for linear programs," Operations Research, vol. 8, no. 1, pp. 101-111, 1960.

[19] G. Brønmo, M. Christiansen, K. Fagerholt, and B. Nygreen, "A multi-start local search heuristic for ship scheduling-a computational study," Computers and Operations Research, vol. 34, no. 3, pp. 900-917, 2007.

[20] J. Añez, T. De La Barra, and B. Pérez, "Dual graph representation of transport networks," Transportation Research Part B: Methodological, vol. 30, no. 3, pp. 209-216, 1996.

[21] L. J. Leblanc, "Algorithm for the discrete network design problem," Transportation Science, vol. 9, no. 3, pp. 183-199, 1975.

[22] L. Zhang, S. Lawphongpanich, and Y. Yin, "Reformulating and solving discrete network design problem via an active set technique," in Proceedings of the 18th International Symposium on Transportation and Traffic Theory, W. H. K. Lam, S. C. Wong, and H. K. Lo, Eds., pp. 283-300, Springer, Hong Kong, 2009.

[23] S. Wang, Q. Meng, and H. Yang, "Global optimization methods for the discrete network design problem," Transportation Research Part B: Methodological, vol. 50, pp. 42-60, 2013.

[24] M. Beckmann, C. Mcguire, and C. Winsten, Studies in the Economics of Transportation, Yale University Press, New Haven, Conn, USA, 1956.
[25] M. Aghassi, D. Bertsimas, and G. Perakis, "Solving asymmetric variational inequalities via convex optimization," Operations Research Letters, vol. 34, no. 5, pp. 481-490, 2006.

[26] D. Z. W. Wang and H. K. Lo, "Global optimum of the linearized network design problem with equilibrium flows," Transportation Research Part B: Methodological, vol. 44, no. 4, pp. 482-492, 2010.

[27] H. Farvaresh and M. M. Sepehri, "A single-level mixed integer linear formulation for a bi-level discrete network design problem," Transportation Research Part E: Logistics and Transportation Review, vol. 47, no. 5, pp. 623-640, 2011.

[28] K. G. Murty, Linear Complementarity, Linear and Nonlinear Programming, vol. 3 of Sigma Series in Applied Mathematics, Heldermann, Berlin, Germany, 1988.

[29] J. Nocedal and S. J. Wright, Numerical Optimization, Springer Series in Operations Research and Financial Engineering, Springer, New York, NY, USA, 2nd edition, 2006.

[30] Y. Shafahi and R. Faturechi, "A new fuzzy approach to estimate the O-D matrix from link volumes," Transportation Planning and Technology, vol. 32, no. 6, pp. 499-526, 2009.

[31] A. Brooke, D. Kendrick, A. Meeraus, and R. Raman, GAMS: A User's Guide, GAMS Development Corporation, Washington, DC, USA, 2005.

[32] L. Zhang and J. Sun, "Dual-based heuristic for optimal cordon pricing design," Journal of Transportation Engineering, vol. 139, no. 11, pp. 1105-1116, 2013. 


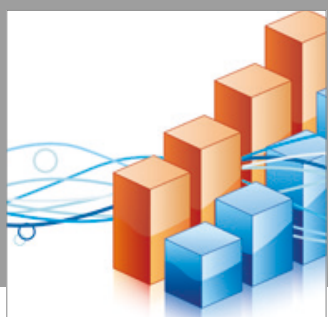

Advances in

Operations Research

vatersals

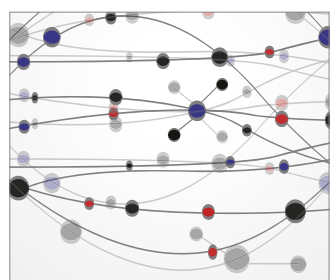

\section{The Scientific} World Journal
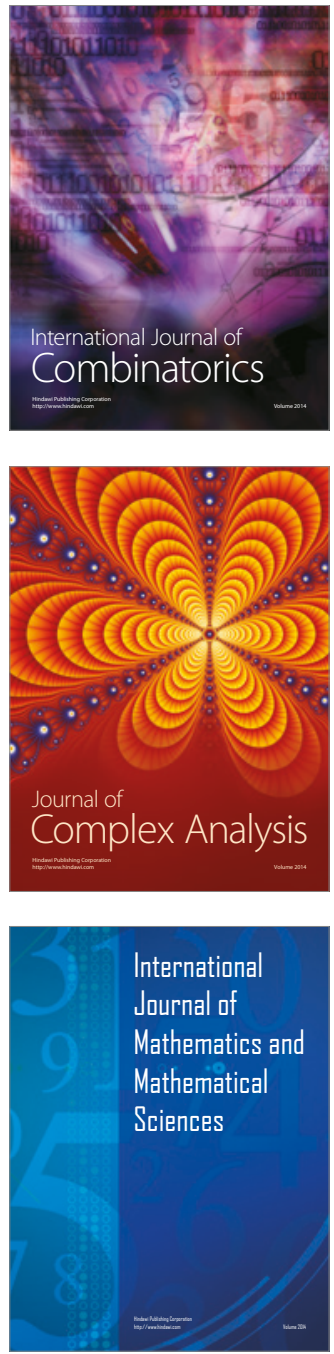
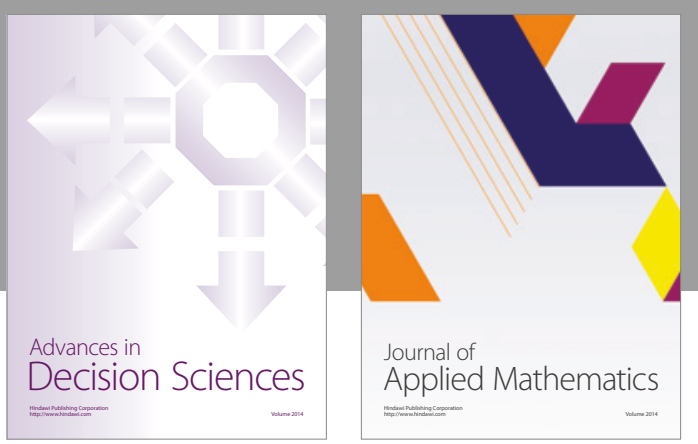

Algebra

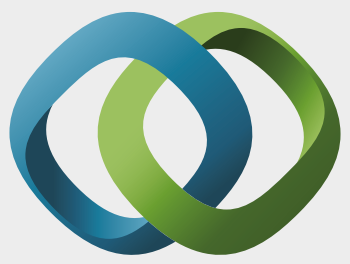

\section{Hindawi}

Submit your manuscripts at

https://www.hindawi.com
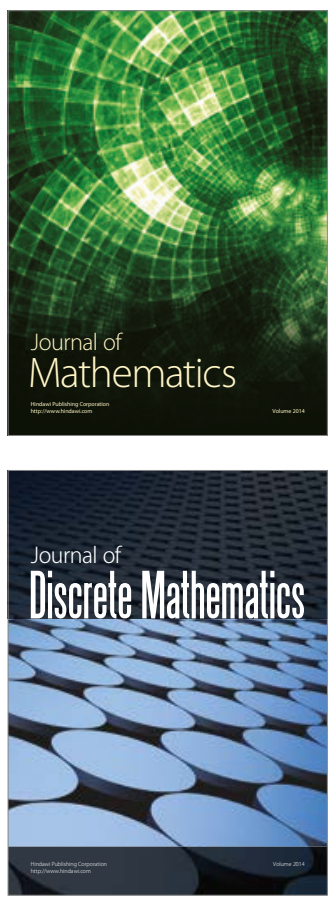

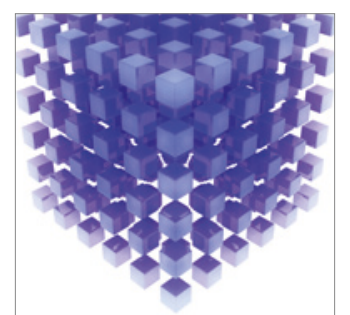

Mathematical Problems in Engineering
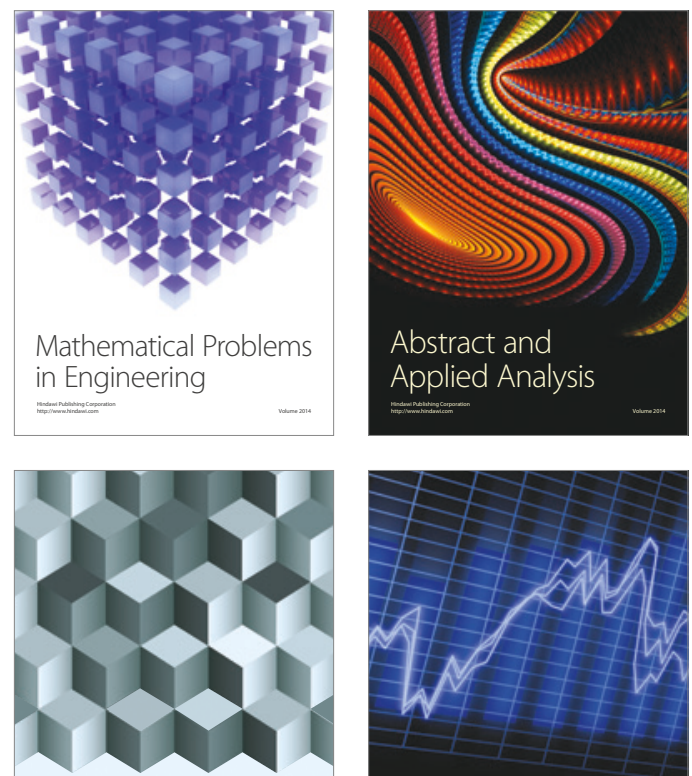

Journal of

Function Spaces

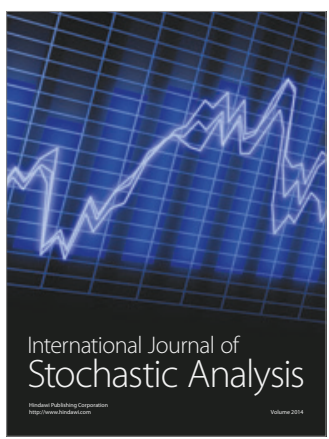

Probability and Statistics
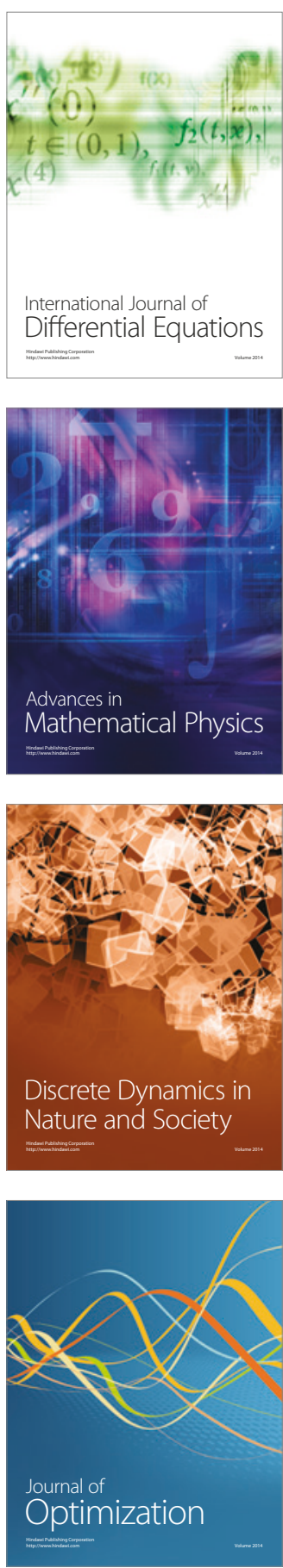